\title{
Antenatal retinoic acid administration increases trophoblastic retinol-binding protein dependent retinol transport in the nitrofen model of congenital diaphragmatic hernia
}

\author{
Balazs Kutasy' ${ }^{1}$ Florian Friedmacher', Lara Pes², David Coyle'1, Takashi Doi' ${ }^{1}$ Francesca Paradisi and Prem Puri'
}

BACKGROUND: Low pulmonary retinol levels and disrupted retinoid signaling pathway (RSP) have been implicated in the pathogenesis of congenital diaphragmatic hernia $(\mathrm{CDH})$ and associated pulmonary hypoplasia (PH). It has been demonstrated that nitrofen disturbs the main retinol-binding protein (RBP)-dependent trophoblastic retinol transport. Several studies have demonstrated that prenatal treatment with retinoic acid (RA) can reverse $\mathrm{PH}$ in the nitrofen-induced $\mathrm{CDH}$ model. We hypothesized that maternal administration of RA can increase trophoblastic RBP-dependent retinol transport in a nitrofen model of $\mathrm{CDH}$.

METHODS: Pregnant rats were treated with nitrofen or vehicle on gestational day 9 (D9) and sacrificed on D21. RA was given i.p. on D18, D19, and D20. Retinol and RA levels were measured using high-performance liquid chromatography. Immunohistochemistry was performed to evaluate trophoblastic expression of RBP. Expression levels of the primary RSP genes were determined using quantitative real-time PCR and immunohistochemistry.

RESULTS: Markedly increased trophoblastic RBP immunoreactivity was observed in $\mathrm{CDH}+\mathrm{RA}$ compared to $\mathrm{CDH}$. Significantly increased serum and pulmonary retinol and RA levels were detected in $\mathrm{CDH}+\mathrm{RA}$ compared to $\mathrm{CDH}$. Pulmonary expression of RSP genes and proteins were increased in $\mathrm{CDH}+\mathrm{RA}$ compared to $\mathrm{CDH}$.

CONCLUSION: Increased trophoblastic RBP expression and retinol transport after antenatal administration of RA suggest that retinol-triggered RSP activation may attenuate CDHassociated PH by elevating serum and pulmonary retinol levels.

$\mathbf{T}$ he mortality rate of infants born with congenital diaphragmatic hernia $(\mathrm{CDH})$ remains high despite prenatal diagnosis and improved postnatal treatment strategies (1). The high morbidity and mortality is mainly attributed to pulmonary hypoplasia $(\mathrm{PH})$ and associated persistent pulmonary hypertension (2). Much of the current understanding of the pathogenesis of $\mathrm{PH}$ in $\mathrm{CDH}$ originates from experimental animal studies. Maternal exposure to nitrofen (2,4-dichlorophenyl-p-nitrophenyl ether) in rodents during midgestation results in a high rate of $\mathrm{CDH}$ and associated $\mathrm{PH}$ in their fetuses, which is strikingly similar to the human condition (3).

It is well understood that retinoids-vitamin A and its derivatives-are essential for the morphogenesis of most developing organs and tissues, including the lungs (4). The placenta has a major role in retinol homeostasis in fetal life (5). As the fetus cannot synthesize retinol, it relies on circulating maternal retinol, which reaches the fetus by crossing the maternal-fetal barrier in the placenta (6). Although retinol-binding protein (RBP) is essential for retinol transport within the circulation (7), maternal RBP does not cross the placenta (8). Therefore, in order to enter the fetal circulation, maternal retinol bound to maternal RBP must be released at the maternal-fetal interface. Fetal RBP is produced by the trophoblast and then forms a complex with retinol to be subsequently released into the fetal circulation and delivered to the target organs (8). This expression is tightly controlled by the placental retinol level through the expression of trophoblastic retinoid acid receptors $($ RAR $\alpha)(8)$.

In recent years, disruption of the retinoid signaling pathway (RSP) has been implicated in the pathogenesis of $\mathrm{PH}$ and $\mathrm{CDH}$ in rodents (9) and human newborns with $\mathrm{CDH}$ (10). We have previously shown that serum and pulmonary total retinol levels are significantly decreased in nitrofen-induced hypoplastic lungs during late lung morphogenesis, supporting the hypothesis that reduced retinol levels contribute to the pathogenesis of $\mathrm{CDH}$ (11). In human newborns with $\mathrm{CDH}$, both retinol and its binding protein in the serum, RBP, have been reported to be decreased, whereas maternal levels were comparable between mothers of $\mathrm{CDH}$ patients and mothers of healthy children (10). Further experimental studies have demonstrated that the trophoblastic expression of RBP and RAR $\alpha$ is decreased in rats with nitrofen-induced $\mathrm{CDH}$, indicating that nitrofen disturbs the mobilization of retinol from the placenta into the fetal circulation (12).

Several studies have demonstrated that antenatal treatment with retinoic acid (RA), the active metabolite of retinol, during

'Our Lady's Children's Hospital, National Children's Research Centre, Dublin, Ireland; ${ }^{2}$ Centre for Synthesis and Chemical Biology, School of Chemistry and Chemical Biology, University College Dublin, Dublin, Ireland. Correspondence: Prem Puri (prem.puri@ncrc.ie) 
gestation increases the expression levels of several genes which are key regulators of the RSP, reverting $\mathrm{PH}$ in the nitrofeninduced CDH model (13). It has been shown that maternal administration of RA can lead to it directly crossing the placenta, elevating the fetal serum RA level (14). However, the precise mechanism by which RA acts in the nitrofen-induced $\mathrm{CDH}$ model still remains unclear. We designed this study to test the hypothesis that maternal administration of RA increases trophoblastic RBP-dependent retinol transport, which in turn results in elevated serum and pulmonary retinol levels in the nitrofen model of $\mathrm{CDH}$.

\section{RESULTS}

\section{Fetus Weights}

Administration of nitrofen decreases total body weight and causes placental hypoplasia and $\mathrm{PH}$ in both the affected and contralateral lungs (Table 1; $P<0.05$ ).

Prenatal administration of RA increased significantly both placenta and total body weight in Control+RA compared to controls (Table $1 ; P<0.05$ ). Both lungs as well as placenta and total body weight were significantly higher in the $\mathrm{CDH}+\mathrm{RA}$ group compared with $\mathrm{CDH}$ group (Table 1, $P<0.05)$.

Table 1. Fetus weights

\begin{tabular}{lcccc}
\hline Weight (g) & Control & Control+RA & CDH & CDH+RA \\
\hline Placenta & $0.576 \pm 0.01$ & $0.681 \pm 0.02^{*}$ & $0.442 \pm 0.01^{* * * *}$ & $0.58 \pm 0.07$ \\
Left lungs & $0.054 \pm 0.002$ & $0.059 \pm 0.003$ & $0.025 \pm 0.001^{*, * *}$ & $0.039 \pm 0.01$ \\
Right lungs & $0.078 \pm 0.004$ & $0.085 \pm 0.001$ & $0.051 \pm 0.002^{*, * *}$ & $0.069 \pm 0.002$ \\
Total body & $5.1 \pm 0.08$ & $6.2 \pm 0.1^{*}$ & $4.68 \pm 0.1^{*, * *}$ & $4.98 \pm 0.07$ \\
weight & & & & \\
\hline $\begin{array}{l}\text { In CDH group, pulmonary hypoplasia, hypoplastic placentas, and lower total body } \\
\text { weight were compared with controls (*P }<0.05 \text { vs. control). Both total body and lung }\end{array}$ \\
$\begin{array}{l}\text { weight were significantly higher in the CDH+RA group compared with CDH group } \\
(* * P<0.05 \text { vs. nitrofen+RA). }\end{array}$
\end{tabular}

\section{$\mathrm{CDH}$ in Fetus}

The incidence rate of $\mathrm{CDH}$ was similar in both $\mathrm{CDH}$ and $\mathrm{CDH}+\mathrm{RA}$ group $(77 / 131,58.7$ vs. $73 / 127,57.4 \%)$. In both $\mathrm{CDH}$ and $\mathrm{CDH}+\mathrm{RA}$ group, the left side was the most commonly affected side (57/77, 74\% and 54/73, 73.9\%).

\section{Maternal Serum Retinoid Levels}

There were no differences in the serum total retinoid, retinol, $\mathrm{RA}$, and retinyl-ester (RE) levels between control and nitrofen mothers (Table 2). Maternal administration of RA significantly increased maternal serum total retinoid, RA and RE levels both in control+RA and $\mathrm{CDH}+\mathrm{RA}$ groups (Table 2; $P<0.05$ ), and serum retinoid levels were similar in both the control+RA and $\mathrm{CDH}+\mathrm{RA}$ groups (Table 2). Serum retinol levels were similar in all four groups (Table 2).

\section{Fetal Serum and Pulmonary Retinoid Levels}

As previously demonstrated, we simultaneously detected significantly increased total serum retinoid and decreased serum retinol levels in the $\mathrm{CDH}$ group compared with controls (Table 3; $P<0.05)$ (11). In controls, the primary retinoid present in the serum by proportion was retinol (97\%), whereas in $\mathrm{CDH}, \mathrm{RE}$, at $92 \%$, was proportionately the primary retinoid in the serum (Table 3 ).

Prenatal administration of RA significantly increased fetal serum RA as well as total retinoid, retinol, and RE levels in both control+RA and $\mathrm{CDH}+\mathrm{RA}$ groups compared with controls and $\mathrm{CDH}$ (Table 3; $P<0.05)$. In contrast with the $\mathrm{CDH}$ group, retinol was the main retinoid $(65.8 \%)$ in the serum by proportion in the $\mathrm{CDH}+\mathrm{RA}$ group (Table 3 ).

Previously we demonstrated that both total pulmonary retinoid and pulmonary retinol levels were significantly decreased in the CDH group compared with controls (Table 4) (11). Significantly increased total pulmonary retinoid levels were measured in both control+RA and $\mathrm{CDH}+\mathrm{RA}$ fetuses compared with controls and $\mathrm{CDH}$ (Table $4, P<0.05$ ). The pulmonary

Table 2. Maternal serum retinoid level

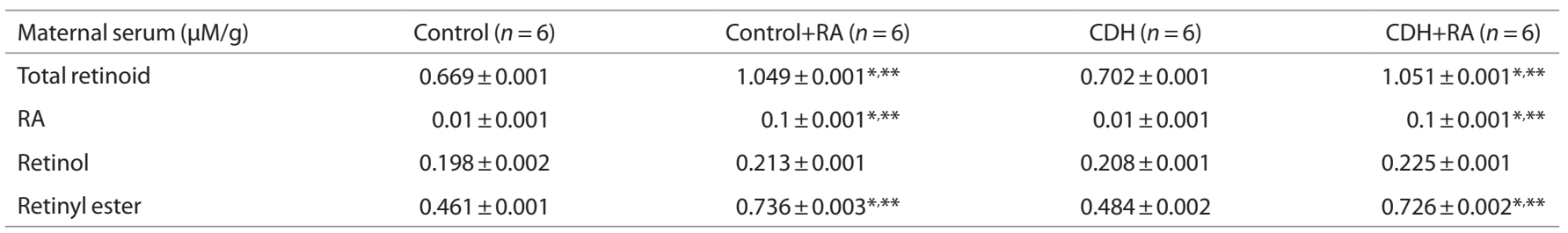

In every group, the maternal serum retinol levels were similar. Prenatal administration of RA increased total retinoid, RA, and retinyl ester level in control+RA and CDH+RA group compared with control and $\mathrm{CDH}\left({ }^{*} P<0.05\right.$ vs. control; ${ }^{* *} P<0.05$ vs. $\left.\mathrm{CDH}\right)$.

Table 3. Fetal serum retinol levels

\begin{tabular}{lccc}
\hline Fetal serum $(\mu \mathrm{M} / \mathrm{g})$ & Control $(n=8)$ & Control+RA $(n=8)$ & $\mathrm{CDH}(n=8)$ \\
\hline Total retinoid & $0.0897 \pm 0.002(100 \%)$ & $0.196 \pm 0.003^{*}(100 \%)$ & $0.198 \pm 0.008^{*}(100 \%)$ \\
RA & $0.0001 \pm 0.001(0.1 \%)$ & $0.001 \pm 0.002^{*, * *}(0.5 \%)$ & $0.0001 \pm 0.001(<0.01 \%)$ \\
Retinol & $0.0872 \pm 0.001(97 \%)$ & $0.181 \pm 0.001^{*, * *}(92 \%)$ & $0.0151 \pm 0.003^{*}(7.6 \%)$ \\
Retinyl ester & $0.00245 \pm 0.002(2.7 \%)$ & $0.014 \pm 0.004^{*, * *}(7.1 \%)$ & $0.1829 \pm 0.005^{*}(92 \%)$ \\
\hline
\end{tabular}

Compared with controls, the total retinoid and retinyl-ester levels were significantly increased; and retinol level was significantly decreased in serum in the CDH group $(* P<0.05$ vs. control). Prenatal administration of RA significantly increases serum level of total retinoid and retinol and significantly decreases retinyl ester level in the $C D H+R A$ group $(* * P<0.05$ vs.

$\mathrm{CDH})$. Retinol was the main retinoid in the serum, both in control and $\mathrm{CDH}+\mathrm{RA}$ groups, whereas in CDH group retinyl ester was the main retinoid in the serum. 


\section{Articles | Kutasy etal.}

RA, retinol, and RE levels were significantly increased in the $\mathrm{CDH}+\mathrm{RA}$ group compared with $\mathrm{CDH}$ (Table $4, P<0.05$ ).

\section{Immunohistochemical Staining of Trophoblastic RAR $\alpha$ and RBP}

We have shown earlier that nitrofen markedly decreases trophoblastic RAR $\alpha$ and RBP expression (Figures 1 and 2) (11). Prenatal administration of RA markedly increased trophoblastic RAR $\alpha$ and RBP immunoreactivity in control+RA and $\mathrm{CDH}+\mathrm{RA}$ placentas compared with control and $\mathrm{CDH}$ (Figures 1 and 2).

\section{Total Pulmonary Protein Level of RBP}

In addition to the finding of reduced trophoblastic RBP expression, we also detected decreased pulmonary RBP levels in the $\mathrm{CDH}$ group compared with controls (11). In left lungs, significantly increased total RBP protein levels were detected in the $\mathrm{CDH}+\mathrm{RA}$ group compared with $\mathrm{CDH}$ (Table $5 ; P<0.05$ ).

\section{Pulmonary RSP Gene Expression}

The relative mRNA expression levels of cellular retinol-binding protein-1 (CRBP1), retinol dehydrogenase-1a3 (RALDH1a3), retinoic acid receptor $\alpha(R A R \alpha)$, retinoic acid receptor $\beta$ $(R A R \beta)$, and retinoic $X$ receptor $\alpha(R X R \alpha)$ genes were significantly upregulated in $\mathrm{CDH}$ lungs compared with controls (Table 6) (9).

The relative pulmonary mRNA expression levels of main components of RSP genes were significantly increased in control+RA compared with controls and in the $\mathrm{CDH}+\mathrm{RA}$ group compared with $\mathrm{CDH}$ (Table $6 ; P<0.05$ ). Immunohistochemistry showed that the number of alveoli was decreased in $\mathrm{CDH}$ compared with controls (13). We observed that the number of alveoli was markedly increased in $\mathrm{CDH}+\mathrm{RA}$ group compared with $\mathrm{CDH}$. Markedly increased immunoreactivity of RSP was observed in control+RA and $\mathrm{CDH}+\mathrm{RA}$ lungs compared with control and $\mathrm{CDH}$ (Figure 3).

Table 4. Fetal pulmonary retinol levels

\begin{tabular}{|c|c|c|c|c|}
\hline Fetal pulmonary $(\mu \mathrm{M} / \mathrm{g})$ & Control $(n=8)$ & Control+RA $(n=8)$ & $\mathrm{CDH}(n=8)$ & $\mathrm{CDH}+\mathrm{RA}(n=8)$ \\
\hline Total retinoid & $0.7772 \pm 0.05$ & $0.874 \pm 0.03^{*}$ & $0.5133 \pm 0.01^{*}$ & $0.978 \pm 0.02^{*, * *}$ \\
\hline RA & $0.0001 \pm 0.001$ & $0.001 \pm 0.003^{*, * *}$ & $0.0001 \pm 0.001$ & $0.001 \pm 0.003^{*, * *}$ \\
\hline Retinyl ester & $0.69 \pm 0.03$ & $0.75 \pm 0.02^{*}$ & $0.44 \pm 0.02^{*}$ & $0.79 \pm 0.04^{* * * *}$ \\
\hline
\end{tabular}

Both total retinoid and retinol levels were significantly decreased in lungs in $C D H$ compared with controls $(* P<0.05 \mathrm{vs}$. control). Prenatal administration of RA increases both total retinoid and retinol levels in left lungs in control+RA and CDH+RA compared with control (*P<0.05 vs. control) and $C D H\left({ }^{* *} P<0.05\right.$ vs. CDH).
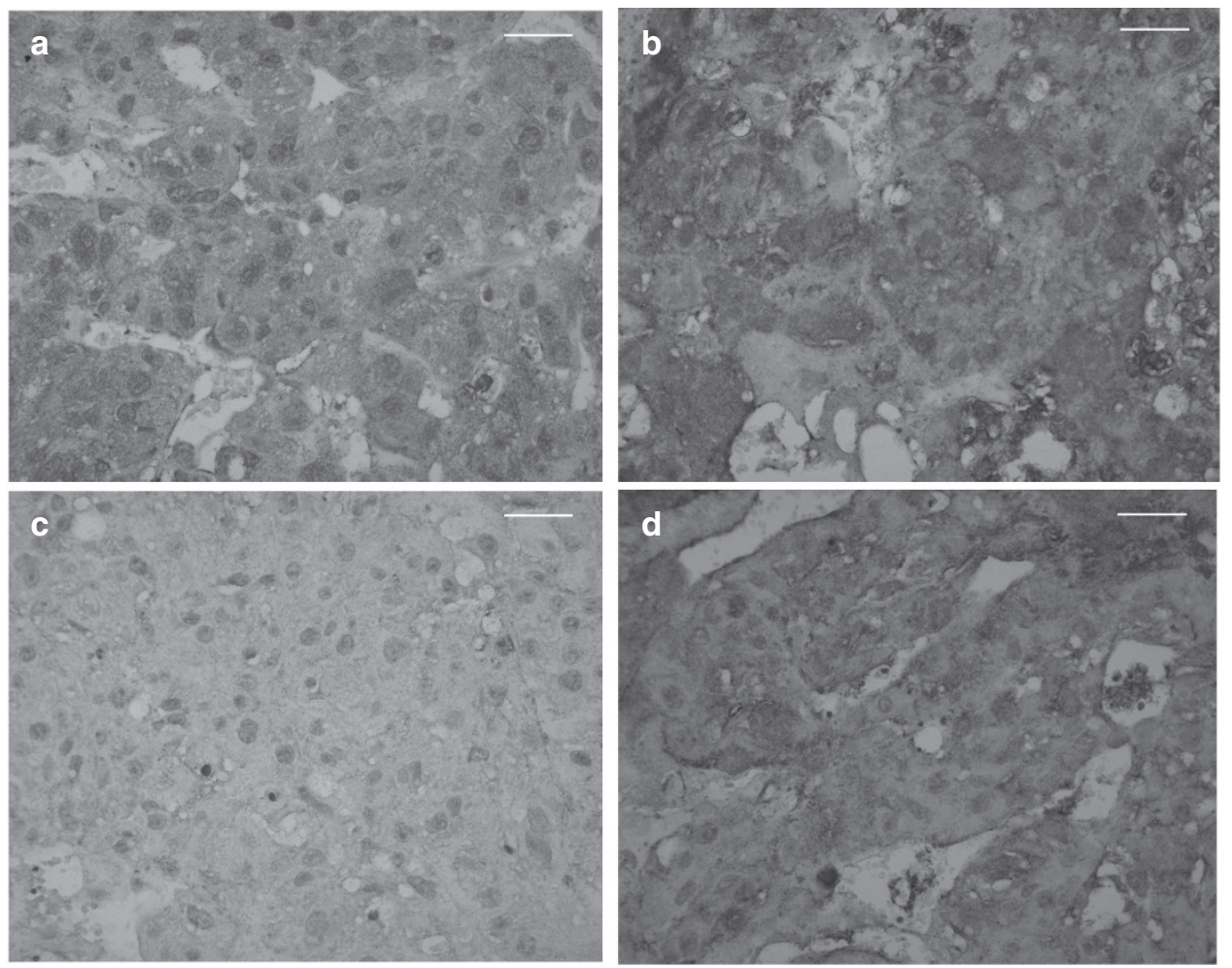

Figure 1. Trophoblastic RAR $\alpha$ expression. Trophoblastic RAR $\alpha$ expression on $\times 20$ magnification. (Bar $=1 \mathrm{~nm}$. (a) control, (b) control+RA, (c) $\mathrm{CDH}$, (d) $\mathrm{CDH}+\mathrm{RA}$ ). (c) Nitrofen markedly decreases trophoblastic RAR $\alpha$ immunoreactivity. Prenatal administration of RA markedly increases trophoblastic RAR $\alpha$ immunoreactivity in (b) control+RA and (d) $\mathrm{CDH}+\mathrm{RA}$ group compared with (a) control and (c) $\mathrm{CDH}$. 

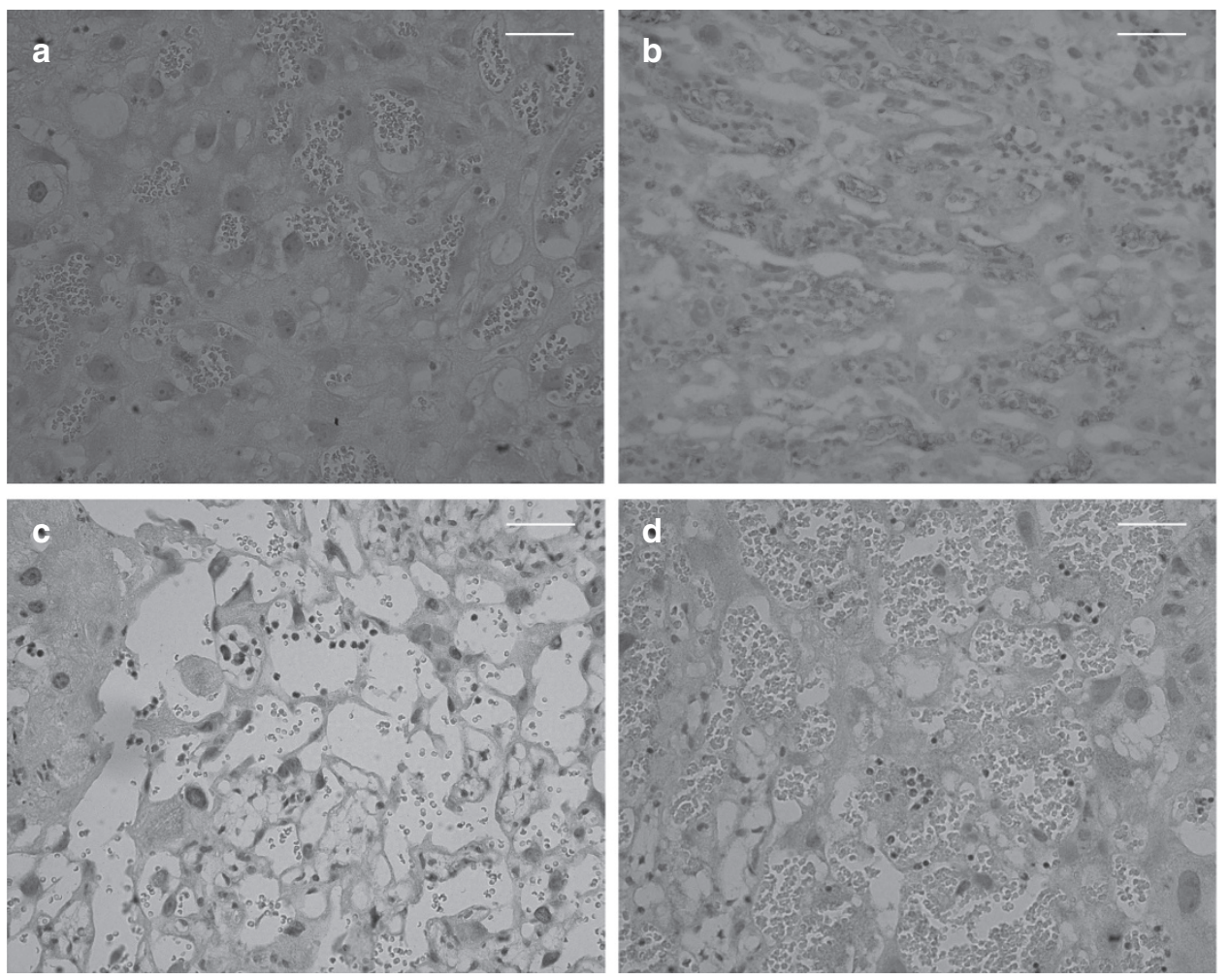

Figure 2. Trophoblastic RBP expression. Trophoblastic RBP expression on $\times 20$ magnification. (Bar $=1 \mathrm{~nm}$. (a) control, (b) control+RA, (c) $\mathrm{CDH}$, (d) $\mathrm{CDH}+\mathrm{RA}$ ). (c) Nitrofen markedly decreases trophoblastic RBP immunoreactivity. Prenatal administration of RA markedly increases trophoblastic RBP immunoreactivity in (d) $\mathrm{CDH}+\mathrm{RA}$ group compared with (a) control and (c) $\mathrm{CDH}$.

Table 5. Pulmonary RBP levels

\begin{tabular}{lcccc}
\hline & Control & Control+RA & CDH & CDH+RA \\
\hline $\begin{array}{l}\text { Pulmonary RBP } \\
\text { level (ng/ml) }\end{array}$ & $409 \pm 0.01$ & $421 \pm 0.02^{*, * *}$ & $260 \pm 0.003^{*}$ & $379 \pm 0.012^{* *}$ \\
\hline & & & &
\end{tabular}

In addition to the reduced trophoblastic RBP expression, the pulmonary RBP level was significantly lower in $\mathrm{CDH}$ compared with controls $(* P<0.05$ vs. control). The pulmonary RBP level was significantly increased in CDH+RA compared with CDH $\left({ }^{*} P<0.05\right.$ vs. $(D H)$.

\section{DISCUSSION}

The nitrofen-induced $\mathrm{CDH}$ model is one of the most widely used animal models to study the pathogenesis of $\mathrm{PH}$ in $\mathrm{CDH}(9,13,15)$. However, the exact molecular mechanism underlying nitrofeninduced PH in this model is still not fully understood. It is known that retinoids, especially retinol, play a key role during fetal lung morphogenesis (4). It has been shown that retinol deficiency alone can induce $\mathrm{PH}$ (16). Because de novo synthesis of retinol does not occur, the developing mammalian embryo requires retinol from the maternal circulation for normal organ development (16). Retinol normally crosses the placenta via the trophoblast. It has been reported that the placenta buffers retinoid delivery by releasing retinol to the fetus when maternal intake is deficient and by storing it to protect the embryo from a potential toxic excess of maternal retinoids (17). Retinol is bound in a complex with the transport protein RBP (7). Quadro et al. (8) investigated fetal retinoid delivery in mice and found that maternal RBP does not cross the placenta. Hence, they concluded that RBP of embryonic origin plays a key role in distributing retinol to the developing tissues (16). It has been reported that the liver is the main source of RBP in adults, whereas fetal RBP levels are mainly dependent on trophoblastic RBP synthesis (16). It has been found in RBPknockout mice that, when RBP-dependent retinol transfer is not working, the retinol is transferred through the placenta alternatively with RE RBP-independent retinol transfer (7).

At a cellular level, target cells take up the retinol-RBP complex or alternatively RE, and retinol is bound to CRBP (18). The CRBP-retinol complex can either be esterified to RE for storage or act as the substrate in the metabolic pathway which leads to the production of the biologically active metabolite, RA by retinol dehydrogenase (RALDH) (9). RA exerts its biological effects through binding to nuclear receptors, the retinoic acid receptors (RARs) and retinoid X receptors (RXRs) (18). RA signaling is tightly regulated by a negative feedback mechanism: elevated RA suppresses the production of CRBP which in turn downregulates the activation of RARs and RXRs (18).

Several authors have shown that the RSP is disrupted in both human and animal models of $\mathrm{CDH}$, and decreased serum and pulmonary retinol levels have been associated with the development of PH in CDH (9-11). The importance of the RSP during fetal development and especially in lung morphogenesis is further supported by a tissue culture study showing that RA administration attenuates the development of $\mathrm{PH}$ in the nitrofen-induced CDH model (13). Beurskens et al. (10) have found decreased serum RBP and retinol levels in human newborns with $\mathrm{CDH}$ compared with controls, whereas mothers of both have comparable levels of RBP and retinol. These authors 


\section{Articles $\mid$ Kutasyet al.}

Table 6. Pulmonary RSP genes mRNA expression levels

\begin{tabular}{|c|c|c|c|c|c|}
\hline & CRBP1 & RALDH1a3 & RAR $\alpha$ & RAR $\beta$ & $\operatorname{RXR} \alpha$ \\
\hline Control $(n=8)$ & $0.78 \pm 0.09$ & $0.03 \pm 0.01$ & $0.54 \pm 0.04$ & $0.08 \pm 0.01$ & $0.14 \pm 0.01$ \\
\hline Control+RA $(n=8)$ & $1.52 \pm 0.07^{*, * *}$ & $0.04 \pm 0.01^{*, * * *}$ & $0.94 \pm 0.04^{*, * *}$ & $0.13 \pm 0.01^{*, * * *}$ & $0.38 \pm 0.01^{* * * *}$ \\
\hline $\mathrm{CDH}+\mathrm{RA}(n=8)$ & $1.71 \pm 0.10^{*, * *}$ & $0.10 \pm 0.01^{*, * *}$ & $1.22 \pm 0.08^{*, * *}$ & $0.19 \pm 0.01^{*, * *}$ & $0.48 \pm 0.03^{*, * *}$ \\
\hline
\end{tabular}

RSP genes were significantly upregulated in $C D H$ compared with controls $(* P<0.05$ vs. control). The relative mRNA expression levels of RSP genes were significantly increased in control+RA compared with control $(* P<0.05$ vs. control) and in CDH+RA compared with $C D H(* * P<0.05$ vs. CDH).

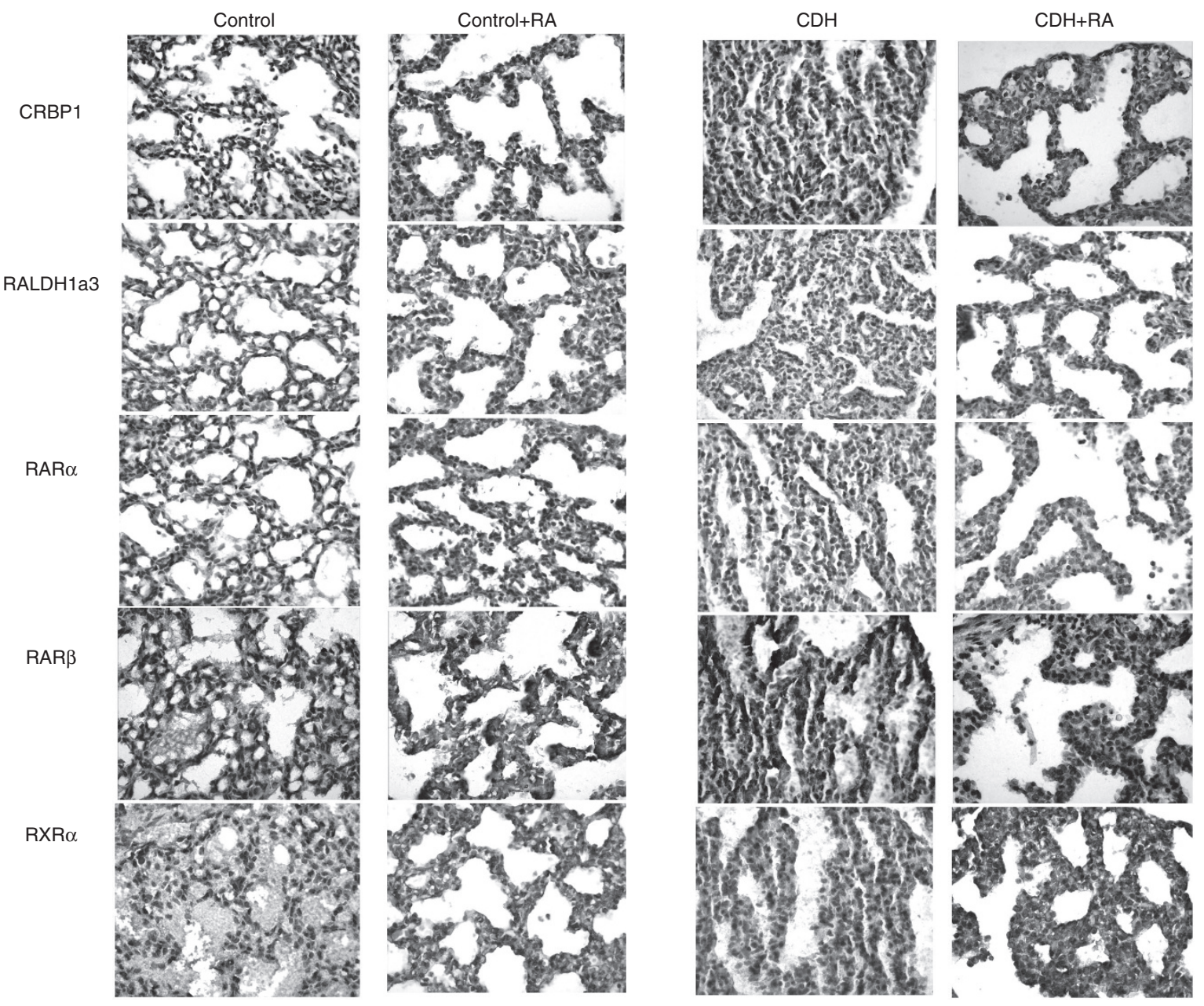

Figure 3. Immunohistochemistry of retinoid signaling pathway expression in lungs. Expression of the main component of RSP in fetal lungs on $\times 20$ magnification. The number of alveoli was decreased in $\mathrm{CDH}$ compared with controls. Prenatal treatment with RA promoted pulmonary alveogenesis. Immunoreactivity of RSP was markedly increased in $\mathrm{CDH}+\mathrm{RA}$ lungs compared with $\mathrm{CDH}$.

concluded that the maternal-fetal transport may be disrupted, thus leading to $\mathrm{CDH}$ and associated $\mathrm{PH}$.

Recently, we showed that nitrofen does not disturb the maternal-placental retinol transfer, but it disturbs somehow the RBPdependent retinol mobilization from placenta to the fetus (19). That results in a high placental retinol level, which, through negative feedback, can decrease trophoblastic RAR $\alpha$ and then RBP expression resulting in decreased pulmonary RBP and retinol level (19). In the circulation, retinol primarily exists in retinolRBP formation, although at lower concentrations other forms of retinol circulate in the bloodstream such as RE incorporated in lipoprotein particles and RA (6). It has been demonstrated that nitrofen does not disturb the alternative, RBP-independent RE maternal-fetal retinol transport. This RE pathway is the main source of retinol during lung morphogenesis in the nitrofen $\mathrm{CDH}$ model (11). Tzimas et al. (14) have investigated the serum RA distribution in both maternal serum and in the embryo. They gave different doses of s.c. RA to the mother rats and measured the dams' RA levels. They found that embryonic/maternal concentration ratio of RA was similar after different doses of RA and that embryonic RA distribution was dependent on the maternal RA concentration. Several studies have demonstrated that 
maternal i.p. administration of RA during gestation increases the expression levels of several genes which are key regulators of the RSP, reverting PH in the nitrofen-induced CDH model (13).

In this study, we found that nitrofen did not interfere with the concentration gradient RA maternal-fetal transport. Antenatal administration of RA, the active metabolite of retinol in the control+RA group, can increase trophoblastic RAR $\alpha$ and RBP expression resulting in increased serum and pulmonary retinol levels. The increased trophoblastic RAR $\alpha$ and RBP expression together with increased serum and pulmonary retinol levels in the $\mathrm{CDH}+\mathrm{RA}$ group suggest that nitrofen disturbs the placental-fetal retinol mobilization through trophoblastic RAR $\alpha$ expression. Furthermore, this may also suggest that the activation of RAR $\alpha$ can reverse the nitrofen effect on decreased RBPdependent placental-fetal retinol mobilization. Interestingly, we found that the highest retinol level was in the $\mathrm{CDH}+\mathrm{RA}$ group. That may suggest that nitrofen can potentially interfere (negative feedback) with the regulation of RBP-dependent retinol mobilization resulting in high retinol mobilization and levels.

We observed, concurrently with elevated retinol levels, that prenatal administration of RA in the control+RA group can increase pulmonary RSP gene expression, lung weight, and maturation. Because the serum and pulmonary RA concentrations are relatively minute, even a 10 -fold increase in RA level would not significantly increase the serum and pulmonary retinol levels and total body and lung weight. Previously, it has been shown in tissue culture that exogenous RA can rescue $\mathrm{PH}$ in nitrofeninduced hypoplastic lungs (13). We found that the level of RA to reach the fetal lungs after prenatal administration of RA would be thousands of times lower than the level that would reverse $\mathrm{PH}$ in the tissue culture. We believe that reversing $\mathrm{PH}$ in the live animal model may be attributable to the increased retinol level and not to the increased RA level. Our results show that increased retinol levels in $\mathrm{CDH}+\mathrm{RA}$ group can increase total body weight and pulmonary weight. Therefore, we suggest that enhanced retinol levels accompanied by the increased gene expression of the main components of RSP may cause retinol-triggered RSP activation leading to reversal of $\mathrm{PH}$ in the nitrofen model of $\mathrm{CDH}$.

In our series, the incidence rate of $\mathrm{CDH}$ was similar in both $\mathrm{CDH}$ and $\mathrm{CDH}+\mathrm{RA}$ groups. Because we administrated RA only on D18, D19 and D20, after when the diaphragmatic defect developed in $\mathrm{CDH}$, the increased retinol level could only affect late lung morphogenesis without impacting the incidence of $\mathrm{CDH}$. The concept of a study examining whether early administration of RA alters the incidence of $\mathrm{CDH}$ is an intriguing one.

It is well known that RA is highly teratogenic. Further investigation needs to be performed to determine the lowest RA level which can reverse $\mathrm{PH}$ in this model or what other nontoxic drugs can increase trophoblasstic RAR $\alpha$ expression. In this study, we have used only all-trans RA in one standard concentration, and we have not examined the levels of other RA metabolites such as 13-cis-RA or etertinate.

\section{METHODS}

\section{Animals, Drugs, and Experimental Design}

Eighty-eight adult Sprague-Dawley rats were mated, and females were checked daily for plugging. The presence of spermatozoids in vaginal smear was considered as a proof of pregnancy; the day of observation determined gestational day 0 (D0). Pregnant female rats were then randomly divided into four groups (control, control with RA, nitrofen, and nitrofen with RA). At D9 (term, 22 d), animals in the experimental group received $100 \mathrm{mg}$ of nitrofen (WAKO Chemicals, Osaka, Japan) dissolved in $1 \mathrm{ml}$ of olive oil intragastrically under short anesthesia, whereas those in the control group received only vehicle. Five milligrams per kilo of all-trans RA (Sigma-Aldrich, Steinheim, Germany) were given i.p. under short anesthesia on D18, D19, and D20 in nitrofen with RA group. Fetuses were harvested by cesarean section on D21 and divided into four groups: control, control with RA (control+RA group), nitrofen with $\mathrm{CDH}$ (CDH group), and nitrofen with RA (CDH+RA group). The animals that received nitrofen, and in whom $\mathrm{CDH}$ was not present, were not investigated. Only the animals that had left side $\mathrm{CDH}$ were included in this study, and only left lungs were studied. In the control group 8 out of 107, in the control+RA group 8 out of 104 , in the $\mathrm{CDH}$ group 8 out of 72 , and in the CDH+RA group 8 out of 88 fetuses were randomly chosen for investigation.

The Department of Health and Children approved the protocol of these animal experiments (Ref: B100/4378) under the Cruelty to Animals Act, 1876; as amended by European Communities Regulations 2002 and 2005, and all animals were treated according to the current guidelines of animal care.

\section{Tissue Collection}

After sedation with isoflurane, term fetuses were harvested free from the dams. Placentas and lungs were dissected from each fetus. Maternal and fetal blood was taken by intracardiac puncture for retinol determination. Freshly prepared serum samples for high-performance liquid chromatography (HPLC) were stored in aliquots at $-80^{\circ} \mathrm{C}$ after clotting for $2 \mathrm{~h}$ and centrifugation for $10 \mathrm{~min}$ at $1,000 \mathrm{~g}$. Fetal lung samples for quantitative real-time PCR (qRT-PCR) were kept in a TRIzol reagent (Invitrogen, Carlsbad, CA) and stored at $-20^{\circ} \mathrm{C}$ until further analysis. Fetal lung samples for determination of RBP levels by enzyme linked immunoassay were snap-frozen in liquid nitrogen and then stored at $-80^{\circ} \mathrm{C}$. Placenta and lung samples for immunohistochemistry were fixed in $4 \%$ formalin and embedded in paraffin.

\section{Immunohistochemistry}

The paraffin-embedded fetal lungs and placentas were sectioned at a thickness of $5 \mu \mathrm{m}$, deparaffinized with xylene, and then rehydrated through ethanol and distilled water. Tissue sections were immersed in target retrieval solution (DAKO Ltd, Cambridgeshire, UK) heated for $10 \mathrm{~min}$ at $121{ }^{\circ} \mathrm{C}$ followed by incubation in $0.03 \% \mathrm{H}_{2} \mathrm{O}_{2}$ for $30 \mathrm{~min}$ to block endogenous peroxidase activity. Sections were incubated overnight at $4{ }^{\circ} \mathrm{C}$ with a $1: 100$ dilution of rabbit polyclonal primary antibody against RXR $\alpha$ (sc553; Santa Cruz Biotechnology, Dallas, TX), 1:50 dilution of goat polyclonal primary antibody against RAR $\alpha$

Table 7. Primers for quantitative real-time PCR

\begin{tabular}{|c|c|c|}
\hline Gene & & Sequence \\
\hline \multirow[t]{2}{*}{$\beta$-Actin } & Forward & 5'-TTGCTGACAGGATGCAGAAG-3' \\
\hline & Reverse & 5'-TAGAGCCACCAATCCACACA-3' \\
\hline \multirow[t]{2}{*}{ CRBP1 } & Forward & $5^{\prime}$-AGTGCATGACCACAGTGAGC-3' \\
\hline & Reverse & 5'-ACCCTCTGCTCTCATCTCCA-3' \\
\hline \multirow[t]{2}{*}{ RALDH1A3 } & Forward & $5^{\prime}$-TCGAGAGTGGGAAGAAGGAA-3' \\
\hline & Reverse & 5'-AGAAGACGGTGGGTTTGATG-3' \\
\hline \multirow[t]{2}{*}{$R A R \alpha$} & Forward & 5'-CCTGCCTCGAATCTACAAGC-3' \\
\hline & Reverse & 5'-GATACTGAGTCGGAAGAAGC-3' \\
\hline \multirow[t]{2}{*}{$R A R \beta$} & Forward & $5^{\prime}$-CCAGGTATACCCCAGAGCAA-3' \\
\hline & Reverse & $5^{\prime}$-GTCAGTCAGAGGACCGAAGC-3' \\
\hline \multirow[t]{2}{*}{$R X R \alpha$} & Forward & 5'-GGTACTTCGTGGGGTCTTCA-3' \\
\hline & Reverse & $5^{\prime}$-TGGGGGTACTCCAAACAGAGG-3' \\
\hline
\end{tabular}


(ab28767, Abcam, Cambridge, UK), 1:50 dilution of goat polyclonal primary antibody against RALDH1A3 (sc26713; Santa Cruz Biotechnology), 1:100 dilution of mouse monoclonal primary antibody against CRBP1 (ab24090; Abcam), 1:100 dilution of primary antibody against RAR $\beta$ (ab53161; Abcam), and 1:100 dilution of mouse monoclonal primary antibody against RBP (sc48384; Santa Cruz Biotechnology). After intensive washing, sections were incubated with corresponding horseradish peroxidase-labeled secondary antibodies and then processed using a DAKO EnVision kit (DAKO), developed with a diaminobenzidine- $\mathrm{H}_{2} \mathrm{O}_{2}$ substrate complex, and counterstained with hematoxylin.

\section{HPLC analysis}

Preparation of samples for measurement of total retinol concentration (including retinol, RE, retinoid acid, and other metabolites of Vitamin A) was performed by modification of a previously described protocol (9). Total retinol concentrations of fetal lungs and fetal and maternal serum were analyzed by HPLC provided with SPD-10A Shimadzu UV-Vis detector on a $3.9 \times 150 \mathrm{~mm}, 5-\mu \mathrm{m}$ reverse phase Resolve C18 column (Waters, Milford, MA). The elution phase was acetonitrile/methanol/ dimethylsulfoxide (90:10:1), and the flow rate was $1.0 \mathrm{ml} / \mathrm{min}$. Chromatograms were extracted at a wave lengths of $325 \mathrm{~nm}$. The concentration of each sample was extrapolated from a calibration curve obtained with pure retinol samples (Sigma-Aldrich) between 0.1 and $10 \mu \mathrm{g} / \mathrm{ml}$ in concentration.

Preparation of samples for simultaneous determination of retinol, $\mathrm{RE}$, and RA was performed by modification of a previously described protocol (20). Retinol, RE, and RA concentrations of serum and lungs were analyzed by HPLC as described above.

\section{RNA Isolation and qRT-PCR}

Total RNA of fetal lungs was isolated using a TRIzol reagent (Invitrogen) according to the manufacturer's protocol. Quantification of total RNA was performed spectrophotometrically (NanoDrop ND-1000 UV-Vis Spectrophotometer). Total RNA (1 $\mu \mathrm{g})$ was reversetranscribed using a Transcriptor High Fidelity cDNA Synthesis Kit (Roche Diagnostics, West Sussex, UK) according to the manufacturer's instruction. Following reverse transcription at $44^{\circ} \mathrm{C}$ for $60 \mathrm{~min}$, qRT-PCR was performed using a LightCycler 480 SYBR Green I Master (Roche Diagnostics) according to the manufacturer's protocol. Gene-specific primer pairs are listed in Table 7 (9). After an initial denaturation step of $5 \mathrm{~min}$ at $95^{\circ} \mathrm{C}, 45$ cycles of amplification for each primer pair were carried out. Each cycle included a denaturation step $\left(10 \mathrm{~s}\right.$ at $\left.95^{\circ} \mathrm{C}\right)$, an annealing step $\left(15 \mathrm{~s}\right.$ at $\left.60^{\circ} \mathrm{C}\right)$, and an elongation step $\left(10 \mathrm{~s}\right.$ at $\left.72^{\circ} \mathrm{C}\right)$. Final elongation temperature was $65^{\circ} \mathrm{C}$ for $1 \mathrm{~min}$. Relative levels of gene expression were measured using a LightCycler 480 (Roche Diagnostics) according to the manufacturer's instructions. The relative changes in the expression levels of CRBP1, $R A L D H 1 A 3, R A R \alpha, R A R \beta$, and $R X R \alpha$ genes were normalized against the level of $\beta$-actin gene expression in each sample. Experiments were carried out at least in duplicate for each data point.

Protein Extraction and Enzyme-Linked Immunoabsorbent Assay The total protein of left lung derived from fetuses was isolated using TRIzol Reagent (Invitrogen), according to the manufacturer's protocol. The obtained protein pellet was dissolved in 1\% SDS, and total protein was dissolved in diethyl pyrocarbonate-treated water. Total protein concentration was determined spectrophotometrically (NanoDrop ND-1000 UV-vis spectrophotometer) by Bradford assay using a quantification kit and bovine serum albumin $(2 \mathrm{mg} / \mathrm{ml})$ as a standard. After extraction, proteins were used for measuring the total RBP (Immunology Consultants Laboratory, Portland, OR) levels by rat ELISA kit according to the manufacturer's protocol. The results were measured at $450 \mathrm{~nm}$ with Synerg Mx microplate reader (BioTek, Vinooski, VT) immediately after adding the stop solution. Experiments were carried out at least in duplicate for each data point.

\section{Statistical Analysis}

All numerical data are presented as mean \pm SEM. Differences between groups at D21 were tested using an unpaired $t$-test or $U$-test, depending on the distribution of data. Statistical significance was accepted at $P$ values $<0.05$.

\section{STATEMENT OF FINANCIAL SUPPORT}

This translational study was supported financially by a grant from National Children's Research Centre, Dublin, Ireland.

Disclosure: None of the authors have disclosure.

\section{REFERENCES}

1. Colvin J, Bower C, Dickinson JE, Sokol J. Outcomes of congenital diaphragmatic hernia: a population-based study in Western Australia. Pediatrics 2005;116:e356-63.

2. Robinson PD, Fitzgerald DA. Congenital diaphragmatic hernia. Paediatr Respir Rev 2007;8:323-34; quiz 334-5.

3. Montedonico S, Nakazawa N, Puri P. Congenital diaphragmatic hernia and retinoids: searching for an etiology. Pediatr Surg Int 2008;24:755-61.

4. Clagett-Dame M, DeLuca HF. The role of vitamin A in mammalian reproduction and embryonic development. Annu Rev Nutr 2002;22:347-81.

5. Wassef L, Quadro L. Uptake of dietary retinoids at the maternal-fetal barrier: in vivo evidence for the role of lipoprotein lipase and alternative pathways. J Biol Chem 2011;286:32198-207.

6. Spiegler E, Kim YK, Wassef L, Shete V, Quadro L. Maternal-fetal transfer and metabolism of vitamin $A$ and its precursor $\beta$-carotene in the developing tissues. Biochim Biophys Acta 2012;1821:88-98.

7. Quadro L, Hamberger L, Colantuoni V, Gottesman ME, Blaner WS. Understanding the physiological role of retinol-binding protein in vitamin A metabolism using transgenic and knockout mouse models. Mol Aspects Med 2003;24:421-30.

8. Quadro L, Hamberger L, Gottesman ME, Colantuoni V, Ramakrishnan R, Blaner WS. Transplacental delivery of retinoid: the role of retinol-binding protein and lipoprotein retinyl ester. Am J Physiol Endocrinol Metab 2004; 286:E844-51.

9. Nakazawa N, Montedonico S, Takayasu H, Paradisi F, Puri P. Disturbance of retinol transportation causes nitrofen-induced hypoplastic lung. J Pediatr Surg 2007;42:345-9.

10. BeurskensLW,TibboelD,LindemansJ, etal.Retinolstatusofnewborninfants is associated with congenital diaphragmatic hernia. Pediatrics 2010;126: 712-20.

11. Kutasy B, Friedmacher F, Pes L, Paradisi F, Puri P. Increased uptake of dietary retinoids at the maternal-fetal barrier in the nitrofen model of congenital diaphragmatic hernia. J Pediatr Surg 2014;49:866-70.

12. Kutasy B, Gosemann JH, Doi T, Fujiwara N, Friedmacher F, Puri P. Nitrofen interferes with trophoblastic expression of retinol-binding protein and transthyretin during lung morphogenesis in the nitrofen-induced congenital diaphragmatic hernia model. Pediatr Surg Int 2012;28:143-8.

13. Montedonico S, Nakazawa N, Puri P. Retinoic acid rescues lung hypoplasia in nitrofen-induced hypoplastic foetal rat lung explants. Pediatr Surg Int 2006;22:2-8.

14. Tzimas G, Nau H, Hendrickx AG, Peterson PE, Hummler H. Retinoid metabolism and transplacental pharmacokinetics in the cynomolgus monkey following a nonteratogenic dosing regimen with all-trans-retinoic acid. Teratology 1996;54:255-65.

15. Kling DE, Cavicchio AJ, Sollinger CA, Schnitzer JJ, Kinane TB, Newburg DS. Nitrofen induces apoptosis independently of retinaldehyde dehydrogenase (RALDH) inhibition. Birth Defects Res B Dev Reprod Toxicol 2010;89:223-32.

16. Quadro L, Hamberger L, Gottesman ME, et al. Pathways of vitamin A delivery to the embryo: insights from a new tunable model of embryonic vitamin A deficiency. Endocrinology 2005;146:4479-90.

17. Sapin V, Chaib S, Blanchon L, et al. Esterification of vitamin A by the human placenta involves villous mesenchymal fibroblasts. Pediatr Res 2000;48: 565-72.

18. Kam RK, Deng Y, Chen Y, Zhao H. Retinoic acid synthesis and functions in early embryonic development. Cell Biosci 2012;2:11.

19. Kutasy B, Pes L, Friedmacher F, Paradisi F, Puri P. Nitrofen increases total retinol levels in placenta during lung morphogenesis in the nitrofen model of congenital diaphragmatic hernia. Pediatr Surg Int 2014;30:1017-22.

20. Moulas AN, Zervos, IA, Taitzoglou IA, Tsantarliotou MP, Botsoglou NA. Simultaneous determination of retinoic acid, retinol, and retinyl palmitate in ram plasma by liquid chromatography. J Liq Chromatogr 2003;26:559-72. 